\title{
Innovación Tecnológica en Empresas Chilenas: Un Estudio Empírico Basado en Patentes
}

\author{
Rodrigo Fuentes Solís ${ }^{1^{*}}$, Sebastián Ferrada Rubio ${ }^{1}$
}

\begin{abstract}
Resumen: La literatura ha centrado sus esfuerzos en estudiar las patentes en determinadas industrias, subsectores o empresas, principalmente en países desarrollados. Por otra parte, el nivel o cantidad de patentes en Chile durante el periodo 2007-2012 en promedio alcanzó las 12 familias triádicas, países como Argentina y México presentaron medias de 12 y 15 familias triádicas respectivamente. La media total para el mismo periodo en la OCDE asciende a 48.242 familias de patentes triádicas. Lo anterior muestra, por una parte el interés de la comunidad científica por estudiar el fenómeno de la creación de patentes, y por otra, la gran brecha tecnológica entre países desarrollados y en vías de desarrollo. Proponemos estudiar los determinantes de la creación de patentes en las empresas chilenas, como un caso de país en vías de desarrollo. Utilizamos un modelo probit en donde la variable dependiente toma valor uno si la empresa tiene o está en proceso de obtener una patente de invención (tecnológica) y cero en caso contrario. Nuestra base tiene datos de 4.338 empresas chilenas, es de corte transversal y corresponde a un extracto de la Octava Encuesta de Innovación en Empresas 2011-2012. Los resultados muestran que la antigüedad de la empresa, la base de conocimiento existente y la utilización de instrumentos gubernamentales de apoyo a la I+D tienen un efecto positivo en la creación de patentes.
\end{abstract}

Palabras claves: Innovación tecnológica; patentes; empresas y probit.

\begin{abstract}
Technological Innovation in Chilean Firms: An Empirical Study Based on Patents. Literature has focused on studying patents in particular industries, subsectors or firms, mainly in developed countries. The level or quantity of patents in Chile during the period 2007-2012 on average reached 12 triadic families, while in countries like Argentina and Mexico presented averages of 12 and 15 respectively. The overall average for the same period amounted to 48,242 OECD triadic patent families. This shows the interest of the scientific community to study the phenomenon of patent creation, and, on the other hand, the large technological gap between developed and developing countries. We propose to study the determinants of patenting in Chilean firms, as a case of a developing country. We use a probit model where the dependent variable takes value one if the firm has or is in the process of obtaining an invention patent (technological) and zero if not. Our database has 4,338 Chilean firms, is crosssectional and corresponds to an extract of the Eighth Survey of Innovation in Firms 2011-2012. The results show that the age of the firm, the base of existing knowledge, and the use of governmental instruments to support R\&D have a positive effect on creation of patents.
\end{abstract}

Keywords: Technological innovation, patents, firms and probit.

Submitted: January $4^{\text {th }} 2016 /$ Approved: October $25^{\text {th }} 2016$

\section{Introducción}

La literatura se ha centrado en estudiar las patentes de invención solo en determinados sectores o rubros específicos. Salmi y Torkkeli (2009) estudian patentes relacionadas con sistemas navegación (GNSS/GPS) en la industria ferroviaria, la base considera 173 datos relacionados a ese subsector. Las patentes van del año 1989 al 2008 y considera principalmente países desarrollados como Japón, Estados Unidos, China, Gran Bretaña entre otros. Respecto a los principales resultados, el número de aplicaciones basadas en GNSS/GPS en la industria ferroviaria es considerablemente menor que para otros medios de trasporte, la actividad de patentamiento fue más alta en el año 2002 (con 24 aplicaciones) y pero ha ido decreciendo en los años recientes. Respecto al país de origen de los inventores, la mayoría (44\%) pertenecía a Japón, demostrando la importancia del tren como medio de transporte y de las investigaciones e innovaciones relacionadas a este.
Hernandez y González (2013) estudian la evolución tecnológica de la industria de celulares a partir de 3 estudios de caso de las empresas Nokia, Huawei y ZTE. Se destaca la actividad de patentamiento como un indicador tangible de la actividad de investigación y desarrollo (I+D). De los principales resultados se destacan dos. Una buena correlación entre las ventas de las empresas y la generación de patentes, sin embargo, no necesariamente esto garantiza el éxito. Por ejemplo Nokia, que a pesar de invertir en $\mathrm{I}+\mathrm{D}$ no tuvo utilidades en los últimos periodos. Segundo, si una empresa sigue el comportamiento del líder (patentamiento), sería factible que llegue a ser líder en el futuro, como es el caso de ZTE.

Tseng y Raudensky (2014) analizan la actividad de patentamiento en las oficinas de transferencia tecnológica de las 20 mayores universidades de EE.UU. De los principales resultados se destacan dos. Primero, que la innovación en el sector universitario e industrial es clave para el incremento de la competitividad. Segundo, la transferencia tecnológica en ha conducido a un crecimiento económico en EE.UU y de seguro conducirá a un próspero futuro en el país.

(1) Escuela de Administración y Negocios, Universidad de Concepción, campus Chillán, Chile. *Corresponding author: rodrfuentes@udec.cl 
Se puede apreciar como la literatura se ha centrado en estudiar las patentes focalizando sus esfuerzos en determinadas industrias, subsectores o empresas y principalmente en países desarrollados. Nuestro trabajo estudia las patentes para el caso chileno, que es un país en vías de desarrollo, e incluimos variadas industrias en nuestra base de datos.

El objetivo de nuestra investigación es determinar que variables influyen en la creación de patentes para el caso chileno. En la sección 2 exponemos las principales motivaciones y contexto del tema. Luego, en la sección 3 presentamos una revisión de la literatura principalmente focalizada en las variables que influirían en la creación de patentes según diversos autores. Posteriormente, en la sección 4, mostramos la metodología utilizada. En la sección 5, presentamos los datos usados, éstos provienen de la Octava Encuesta de Innovación en Empresas (en adelante encuesta de innovación). En la sección 6 estimamos un modelo probit que nos permite estimar la probabilidad de que una empresa decida inscribir una patente de invención y relacionarla con el conjunto de variables que la literatuera sugiere que influyen en este tipo de decisión. En la sección 7 se expone una discusión de los resultados comparando nuestras estimaciones con la litertura revisada. Finalmente, en la sección 8 algunas recomendaciones.

\section{Motivación y contexto}

El uso de patentes es una de las maneras en que las empresas se apropian de los beneficios de una invención (Juliao, Barrios, Schmutzler, \& Sánchez, 2013). Las patentes en Chile, durante el periodo 20072012, en promedio alcanzaron las 12 familias triádicas, por su parte países como México y Argentina presentaron medias de 15 y 12 familias triádicas respectivamente. Por otra parte la media total para el mismo período en la Organización para la Cooperación y el Desarrollo Económico (OCDE) asciende a 48.242 familias de patentes triádicas (OCDE, 2015). Lo anterior muestra, la gran brecha tecnológica entre países desarrollados y en vías de desarrollo.

En cuanto al modo de contabilizar las patentes por la OCDE, estas son medidas mediante familias de patentes triádicas, que son un conjunto de solicitudes de éstas licencias que se presentan ante la European Patent Office (EPO), la Japan Patent Office (JPO) y concedidas por la United States Patent and Trademark Office (USPTO). El motivo de contabilizar este tipo particular de patentes es que permite mejorar la comparabilidad internacional de los indicadores basados en patentes (se comparan sólo entre las solicitudes en un mismo conjunto de países), además que el valor de las patentes suele ser mayor, dado que ampliar la cobertura a las demás naciones presupone un mayor costo, que no retribuiría si la licencia no es lo suficientemente ventajosa (OCDE, 2009).
Gráfico 1. Número de patentes triádicas miembros de la OCDE, año 2012

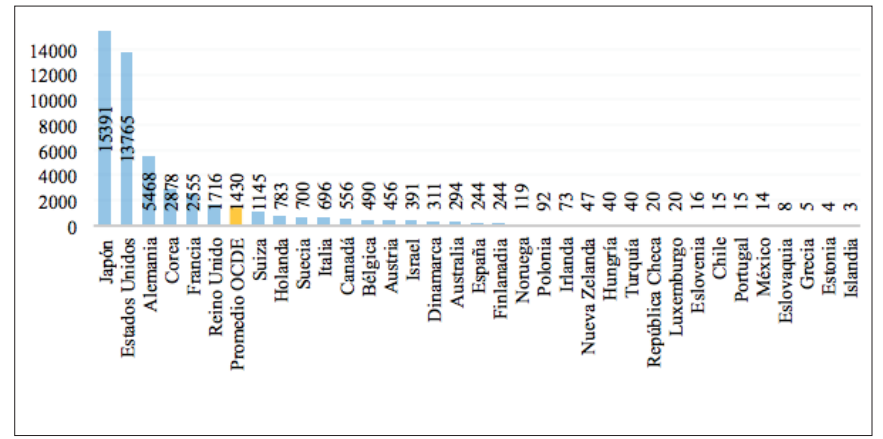

Fuente: Elaboración propia en base a datos de OCDE, 2015.

Tal como se observa en el gráfico anterior, Latinoamérica presenta un evidente rezago frente al resto de las naciones miembros de la OCDE, en la generación de innovación y nuevas tecnologías. Se puede deducir que la mayoría de las firmas a nivel latinoamericano, basan sus procesos de innovación en la imitación, adaptación e ingeniería inversa de métodos y tecnologías de empresas foráneas (Forero, Laureiro, \& Marín, 2011).

La búsqueda del desarrollo es una cuestión importante, es por esto que la innovación, como fuente de crecimiento económico toma un rol fundamental, debido a que sin avance en ciencia y tecnología las naciones se estancan y pierden competitividad frente a otras que sí progresan. Existen investigaciones que buscan entender mejor las variables que influyen en estos procesos, tales como los de Buesa \& Molero (1996), Vega, Fernandez, Gutiérrez, \& Manjarres (2005), y para el caso chileno Benavente (2005), entre otros, los cuales serán profundizados más adelante.

\section{Revisión de literatura}

Las patentes son instrumentos que están vigentes en nuestro mundo desde el siglo XIX, particularmente desde el año 1833. Es en la ciudad de París donde se comienza a resguardar de manera sólida el conocimiento de las personas de ciertas regiones, las cuales ahora cuidarían de sus ideas frente a otros seres que pudieran sacar ventaja de lo ajeno, todo esto sellado en el Convenio de París. La patente es por tanto la forma más antigua de protección a los activos intangibles (Díaz, 2008).

Con el paso de los años surgen en todos los países nuevas y más formas de patentamiento de ideas o invenciones de propiedad industrial como patentes asociadas a procesos, mecanismos, nuevos fármacos, procedimientos, productos, entre otros, las que están relacionadas a muchas áreas tales como, minería, agricultura, pesca, silvicultura, entre otras. 
Hoy en día la competitividad entre las distintas firmas es cada vez más fuerte en el mundo, presentando un escenario hipercompetitivo. Frente a esta situación el patentamiento es primordial, con el fin de protegerse de la copia o la imitación.

Los problemas del cambio tecnológico han ocupado un lugar importante en el análisis económico, una de las preocupaciones básicas ha consistido en buscar indicadores claves para medir las actividades tecnológicas y su relación con la eficiencia económica. Una de estas mediciones se hace a través del número de patentes generados por las empresas (Buesa \& Molero, 1992). Es así como durante el siglo XX y hasta nuestros días la patente se considera un factor determinante de la innovación.

\subsection{Evidencia Empirica}

En este apartado, se recogen en diversas contribuciones teóricas y empíricas para establecer el marco de análisis del patentamiento en las empresas de la industria chilena. La búsqueda de los factores detonantes de la innovación no es algo nuevo, desde Schumpeter a comienzos del siglo XX la innovación ha sido un objetivo de análisis de múltiples autores.

Martínez \& Pérez (2001), identifican que las patentes están relacionadas con la aglomeración de actividades tecnológicas. Esto se refiere a las relaciones que establecen las empresas con instituciones de investigación como las universidades, para acceder a instalaciones de I+D y mano de obra calificada pertenecientes a éstas. Se estima que esta relación es importante en fases iniciales, pero pierde relevancia con el tiempo y el avance de la empresa. Los mismos autores anteriores, definen el uso de tecnologías de la información como la creación de redes o nexos que desarrollan ciertas empresas ya sea dentro de la misma o con otras formando clusters para traspasar información o recursos entre estas. Estos recursos son necesarios para invertirlos conjuntamente en la creación de nuevos conocimientos ( $\mathrm{I}+\mathrm{D}$, diseño, ingeniería), y para la introducción externa de nuevo conocimiento mediante la adquisición, adaptación e implementación de innovaciones (Belussi \& Arcangeli, 1998).

Por otra parte, la relación entre el gasto en I+D realizado por la empresas y las patentes es un tema ampliamente estudiado por la literatura, la que permite hacer ciertas conclusiones con respecto a esta. El gasto en I+D está estrechamente relacionado a las actividades de patentamiento, y además, esta inversión tiene un efecto casi inmediato en la innovación y por tanto en la generación de patentes (Buesa \& Molero, 1992).

De igual forma (Buesa \& Molero, 1992), establecen que el origen del capital o propiedad de la empresa es un factor que incide en la generación de patentes de manera indirecta. Estos autores establecen que empresas de propiedad foránea tienden a importar tecnología por medio de pagos de asistencia técnica y regalías, promoviendo de manera indirecta el patentamiento.

El tamaño de la firma es una variable que ha despertado cierto nivel de interés en la literatura (Rothwell \& Dogson, 1994). Las investigaciones han decantado en dos áreas de debate. La primera estipula que el tamaño de la firma afecta positivamente el desarrollo de patentes, dado que las grandes empresas tienen mayor capacidad para conseguir financiamiento, gestionar la información, mantener grandes instalaciones de $\mathrm{I}+\mathrm{D}$ y atraer a los mejores técnicos y científicos. La segunda área de debate establece que las pequeñas empresas, en especial las de alta tecnología presentan una ventaja en el proceso de cambio técnico debido a su mayor flexibilidad para adaptarse a los cambios en el entorno exterior (Martínez \& Pérez, 2001). Dentro del análisis realizado por Buesa \& Molero (1996), se observa que la probabilidad de que las empresas emprendan actividades innovadoras aumenta junto con el tamaño de esta, asociado al volumen de ventas. Pese a esto ambos autores acotan que la probabilidad de innovar es infravalorada en empresas pequeñas que pueden realizar distintas actividades en $\mathrm{I}+\mathrm{D}$, dimensión de la cual no recogen datos.

La edad o antigüedad de una empresa es otra dimensión que se ha relacionado con la innovación. Buesa \& Molero (1996), señalan que los procesos tecnológicos son acumulativos, la disponibilidad de experiencia constituye un factor favorable para la obtención de resultados innovadores que pueden traducirse en patentes, por lo que la edad de la firma sería un factor a favor del patentamiento.

Sancho (2002), habla sobre los indicadores de ciencia y tecnología dentro de los cuales distingue dos conceptos relacionados a los recursos humanos e $\mathrm{I}+\mathrm{D}$, el primero es personal dedicado a la $\mathrm{I}+\mathrm{D}$, es decir, el número de personas total o parcialmente dedicadas a $\mathrm{I}+\mathrm{D}$. Mientras que el segundo es más general y se refiriere a la reserva de personal en I+D incluyendo al personal real, como al potencial. En los estudios de Vega, Fernández, Gutiérrez \& Manjarres (2005) se observa una relación positiva entre la base de conocimiento existente en la organización y la capacidad de absorción de esta, o capacidad de reconocer el valor de una nueva información y asimilarla con fines comerciales. La base de conocimiento es entendida por los autores mencionados como el conjunto de habilidades, conocimientos, y experiencia del que dispone la organización. Puede ser medida en dos dimensiones, la primera se refiere a las competencias del recurso humano y la experiencia de la organización, factor que se puede relacionar con el nivel de formación académica de los trabajadores. La segunda dimensión se refiere a aspectos relacionados con la infraestructura de la organización, específicamente la existencia de un departamento I+D o similar. En un estudio realizado por Álvarez \& García (2012) se encontró que las competencias o habilidades del recurso humano si son significativas en cuanto a la decisión de innovar, por otro lado no encontraron una relación significativa entre la innovación y la presencia de un departamento de I+D.

Otra variable estudiada son las exportaciones (asociadas a las ventas, y por lo tanto al tamaño de la empresa), para Murillo \& Hill de Titto (2006), una empresa que realiza exportaciones tiene mayores incentivos a innovar, dado que tiene contacto con tecnologías extranjeras y debe satisfacer exigencias de otros mercados. Ambos autores consideran también como variable los apoyos gubernamentales, los cuales contribuyen de manera directa al crear y financiar centros de investigación, subsidiar la investigación privada y proteger las leyes de propiedad intelectual. Cohen y Levin (1989) mencionan esta variable como una de las más estudiadas e importantes asociadas a las 
oportunidades tecnológicas. En numerosos sectores económicos, los gobiernos han contribuido a reducir el costo de la innovación por medio de sus propias investigaciones o subsidiando la investigación en el sector privado. Teubal (1996) hace la distinción de dos fases en la empresa, fase infante y fase madura. Él postula que los subsidios debieran ser la forma de implementar políticas de incentivos a la I+D, durante la fase infante de la firma, en dónde se debiera subsidiar la mitad de los costos del proyecto. En el caso de la fase madura Teubal (1996), menciona la posibilidad que el estado reduzca el apoyo. También existen los programas de exención tributaria, que autorizan a las firmas a decidir libremente qué proyectos de investigación y desarrollo desean financiar.

Benavente (2005), menciona que este tipo de fomento a la innovación debe aplicarse a empresas más grandes con importantes portafolios de proyectos en $\mathrm{I}+\mathrm{D}$, dado que empresas pequeñas pueden poseer bajos niveles de ingreso imponible y, aun cuando la exención tributaria sea máxima, no dispondrán de recursos suficientes para invertir en I+D. Bronwyn \& Van Reenen (2000), establecen que en conjunto hay pruebas sustanciales de que el impuesto tiene un efecto en la I+D realizada, mencionando a grandes rasgos que un dólar dedicado a un incentivos fiscal estimula un dólar invertido en I+D. Hay que mencionar que en Chile existe la Ley Incentivo al I+D (Ley N²0.570) la cual tiene por objetivo impulsar la inversión en $\mathrm{I}+\mathrm{D}$ de las empresas, fomentando la innovación y por ende aumentado la productividad de estas.

La última variable identificada es la localización o la región de origen de la entidad. En un estudio sobre el desarrollo de patentes en universidades españolas (Fernández, Otero, Rodeiro, \& Rodríguez 2009), establecen que la localización de la universidad en una región activa puede conferirle ventajas en el desarrollo de propiedad intelectual. Viendo a las universidades como una representación de las empresas se puede extrapolar esta variable a la división regional chilena. La localización o dimensión geográfica dentro del sistema en que está inserta la firma ha sido un importante factor de análisis, en dónde una cantidad no menor de teorías fueron postuladas con el objeto de explicar el desarrollo de la innovación industrial traducida en patentes, centrándose en la arista geográfica del fenómeno, en especial en su concentración y difusión espaciales (Vilalta \& Banda, 2008). Dichas teorías se pueden clasificar en dos clases, aquellas que aplican un enfoque interdisciplinario geográfico-económico y las que aplican una visión netamente geográfica (Vilalta \& Banda, 2008). Dentro de las teorías que aplican un enfoque interdisciplinario geográfico-económico, la más destacable es la que mencionan Vilalta \& Banda (2008), dentro de su investigación sobre esta área en México, encontraron importantes antecedentes, donde establecen que el crecimiento de las inversiones no se da de manera uniforme, sino que se concentra en diversos polos de dedesarrollo e industrias impulsoras. Además, las teorías existentes respecto a la difusión espacial de la información se divide en cuatro tipos: contagio, expansión, relocalización y difusión jerárquica. La primera se refiere a la manera directa, con contacto entre individuos o instituciones. La difusión por expansión se da cuando la propagación de una idea comienza en un lugar de origen y se traslada a otros lugares, pero sin dejar de usarse en el lugar inicial.
Por otro lado, la difusión por relocalización es similar, pero en este caso la propagación sí deja el lugar de origen. Finalmente, la difusión jerárquica sucede cuando la difusión va desde lugares más grandes a lugares más pequeños (Vilalta \& Banda, 2008). En este contexto, Hägerstrand (1953), establece que la difusión de innovaciones tecnológicas tiende a ser contagiosa. En otras palabras, la difusión de innovación tecnológica es un efecto sujeto a la proximidad física entre instituciones. Vilalta \& Banda (2008), mencionan dos reglas de difusión espacial: la innovación tecnológica se difunde de manera contagiosa en función de la distancia y se encuentra concentrada en ciertos lugares.

A continuación presentamos una tabla resumen de la literatura revisada.

Tabla 1: Principales variables identificadas

\begin{tabular}{|c|c|c|}
\hline Dimensión & Variables & Autor(es) \\
\hline \multirow{2}{*}{ Tamaño } & Tamaño & $\begin{array}{l}\text { (Buesa \& Molero, 1996); } \\
\text { (Martínez \& Pérez, 2001) }\end{array}$ \\
\hline & Exportaciones & $\begin{array}{l}\text { (Murillo \& Hill de Titto, } \\
\text { 2006) }\end{array}$ \\
\hline Edad & Antigüedad & (Buesa \& Molero, 1996) \\
\hline \multirow{2}{*}{$\mathrm{I}+\mathrm{D}$} & Personal en I+D & (Sancho, 2002) \\
\hline & Gasto en $\mathrm{I}+\mathrm{D}$ & (Buesa \& Molero, 1992) \\
\hline $\begin{array}{l}\text { Forma de } \\
\text { propiedad }\end{array}$ & Forma de propiedad & (Buesa \& Molero, 1992) \\
\hline $\begin{array}{l}\text { Región } \\
\text { geográfica }\end{array}$ & Región & $\begin{array}{l}\text { (Fernández, Otero, Rodeiro, } \\
\text { \& Rodríguez, 2009); (Vilalta } \\
\text { \& Banda, 2008) }\end{array}$ \\
\hline $\begin{array}{l}\text { Capital hu- } \\
\text { mano }\end{array}$ & Base de conocimiento & $\begin{array}{l}\text { (Vega, Fernandez, Gutiérrez, } \\
\text { \& Manjarres, 2005); (Álvarez } \\
\text { \& García, 2012) }\end{array}$ \\
\hline $\begin{array}{l}\text { Apoyo } \\
\text { gubernamental }\end{array}$ & $\begin{array}{l}\text { Instrumentos guber- } \\
\text { namentales de apoyo } \\
\text { al I+D }\end{array}$ & $\begin{array}{l}\text { (Cohen \& Levin, 1989); (Mu- } \\
\text { rillo \& Hill de Titto, 2006); } \\
\text { (Benavente, 2005) }\end{array}$ \\
\hline \multirow[b]{2}{*}{$\begin{array}{l}\text { Uso de } \\
\text { información }\end{array}$} & $\begin{array}{l}\text { Uso de tecnologías de } \\
\text { la información }\end{array}$ & (Martínez \& Pérez, 2001) \\
\hline & $\begin{array}{l}\text { Nexo con instituciones } \\
\text { educacionales y } \\
\text { aglomeración de activi- } \\
\text { dades tecnológicas }\end{array}$ & (Martínez \& Pérez, 2001) \\
\hline
\end{tabular}

Fuente: Elaboración propia con base en la literatura revisada.

\section{Metodología}

Probit es un modelo de elección discreta o de respuesta cualitativa, que surge para explicar una variable dependiente binaria ( 0 y 1), se decide respecto a un sí o un no. Este es aplicado para estimar la probabilidad de que ocurra un acontecimiento dados los valores de las variables explicativas. Al utilizar una función de distribución normal se da lugar al modelo probit

$$
\operatorname{Prob}(Y=1)=\int_{-\infty}^{\beta^{\prime}} \phi(t) d t=\Phi\left(\boldsymbol{\beta}^{\prime} \mathbf{x}\right)
$$


Donde la función de distribución normal estándar se representa como, $\phi(),. \beta^{\prime}$ representa los parámetros de las variables explicativas, finalmente el último parámetro de la ecuación es, que representa el vector de variables explicativas (Salgado \& Chovar, 2010).

El modelo de probabilidad (Greene, 1999) es un modelo de regresión donde:

$$
\operatorname{Prob}(Y=1)=\int_{-\infty}^{\beta^{\prime}} \phi(t) d t=\Phi\left(\boldsymbol{\beta}^{\prime} \mathbf{x}\right)
$$

Para estimar los parámetros los modelos de elección binaria utilizan el método de máxima verosimilitud, donde cada observación se considera como la realización individual de una variable aleatoria con distribución Bernoulli (es decir, binomial con $n=1$ ). La probabilidad conjunta, o función de verosimilitud, de un modelo con probabilidad de éxito $F\left(\boldsymbol{\beta}^{\prime} \mathbf{x}\right)$ y observaciones independientes es:

$$
\operatorname{Prob}\left(Y_{1}=y_{1}, Y_{2}=y_{2}, \ldots, Y_{n}=y_{n}\right)=\prod_{y_{i}=0}\left[1-F\left(\boldsymbol{\beta}^{\prime} \mathbf{x}_{1}\right)\right] \prod_{y_{i=1}}\left[F\left(\boldsymbol{\beta}^{\prime} \mathbf{x}_{\mathbf{1}}\right)\right]
$$

Se puede reescribir esta fórmula como

$$
L=\prod_{y_{i}=0}^{n}\left[F\left(\boldsymbol{\beta}^{\prime} \mathbf{x}_{i}\right)\right]^{y_{i}}\left[1-F\left(\boldsymbol{\beta}^{\prime} \mathbf{x}_{i}\right)\right]^{1-y_{i}}
$$

Esta es la función de verosimilitud para una muestra de observaciones. Tomando logaritmo se obtiene:

$$
\ln L=\sum_{i=1}^{n}\left[y_{i} \ln F\left(\boldsymbol{\beta}^{\prime} \mathbf{x}_{i}\right)+\left(1-y_{i}\right) \ln \left(1-\ln F\left(\boldsymbol{\beta}^{\prime} \mathbf{x}_{i}\right)\right)\right]^{6}
$$

Las condiciones de primer orden del problema de maximización requieren que:

$$
\frac{\partial \ln L}{\partial \boldsymbol{\beta}}=\sum_{i=1}^{n}\left[\frac{y_{i} f_{i}}{F_{i}}+\left(1-y_{i}\right) \frac{-f_{i}}{\left(1-F_{i}\right)}\right] \mathbf{x}_{i}=0
$$

En esta última ecuación y lo que sigue usaremos el subíndice $i$ para indicar que la función se evalúa en $\boldsymbol{\beta}^{\prime} \mathbf{x}_{\boldsymbol{i}}$. Al seleccionar una forma concreta para $F_{i}$ se obtiene el modelo. A menos que se utilice el modelo de probabilidad lineal, las ecuaciones contenidas en la anterior serán no lineales y habrán de resolverse con un método iterativo.

Para probit, al utilizar la función de distribución normal la función de verosimilitud logarítmica resultante es:

$$
\ln L=\sum_{y_{i}=0} \ln \left[1-\Phi\left(\boldsymbol{\beta}^{\prime} \mathbf{x}_{i}\right)\right]+\sum_{y_{i}=1} \ln \Phi\left(\boldsymbol{\beta}^{\prime} \mathbf{x}_{i}\right)
$$

Al maximizar (calcular las derivadas respecto al vector $\boldsymbol{\beta}$ ) se obtiene que las condiciones de primer orden son:

$$
\frac{\partial \ln L}{\partial \beta}=\sum_{y_{i}=0} \frac{-\phi_{i}}{1-\Phi_{i}} \mathbf{x}_{i}+\sum_{y_{i}=1} \frac{\phi_{i}}{\Phi_{i}} \mathbf{x}_{i}=\sum_{i=1}^{n}\left(\frac{q_{i} \phi\left(q_{i} \boldsymbol{\beta}^{\prime} \mathbf{x}_{i}\right)}{\Phi\left(q_{i} \boldsymbol{\beta}^{\prime} \mathbf{x}_{i}\right)}\right) \mathbf{x}_{i} \sum_{i=1}^{n} \lambda_{i} \mathbf{x}_{i}=0
$$

Donde $q_{i}=2 y_{i}-1$. En este modelo los estimadores de los parámetros pueden calcularse sin ningún problema utilizando el método de Newton. En el modelo probit el cálculo de estos se hace utilizando $\lambda\left(\mathbf{x}_{i}, \boldsymbol{\beta}^{\prime} \mathbf{x}_{i}\right)=\lambda_{i}$, , donde las segundas derivadas pueden obtenerse para cualquier $\mathrm{z}$,
Con ello se obtiene

$$
\frac{d \phi(z)}{d z}=-z \phi(z)
$$

$$
\mathrm{H}=\frac{\partial^{2} \ln L}{\partial \boldsymbol{\beta} \partial \boldsymbol{\beta}^{\prime}}=\sum_{i=1}^{n}-\lambda_{i}\left(1_{i}+\boldsymbol{\beta}^{\prime} \mathbf{x}_{i}\right) \mathbf{x}_{i} \mathbf{x}_{i}^{\prime}
$$

Esta matriz es negativa sea cual sea el valor de $\boldsymbol{\beta}$. Basta con darse cuenta de que la parte escalar de la sumatoria es $1-\operatorname{Var}\left[\epsilon \mid \epsilon<\boldsymbol{\beta}^{\prime} \mathbf{x}\right]$ cuando $\mathrm{y}=1$ y $1-\operatorname{Var}\left[\epsilon \mid \epsilon>-\boldsymbol{\beta}^{\prime} \mathbf{x}\right]$, cuando y $=0$. En los dos casos, la varianza está entre 0 y 1 , por lo que también su complemento estará en este intervalo (Greene, 1999).

La matriz de covarianzas asintótica del estimador de máxima verosimilitud puede estimarse a partir de la inversa del hessiano evaluada en el estimador de máxima verosimilitud.

Por otro lado, es importante observar que los parámetros del modelo, como los de cualquier modelo no lineal, no coinciden con los efectos marginales (Greene, 1999). En general los parámetros son calculados como

$$
\frac{\partial E[y \mid x]}{\partial y}=\left\{\frac{d F\left(\boldsymbol{\beta}^{\prime} \mathbf{x}\right)}{d\left(\boldsymbol{\beta}^{\prime} \mathbf{x}\right)}\right\} \boldsymbol{\beta}=f\left(\boldsymbol{\beta}^{\prime} \mathbf{x}\right) \boldsymbol{\beta}
$$

Donde $f($.$) la función de densidad asociada a la utilizada, en caso de$ un probit, la distribución normal $\phi($.

$$
\frac{\partial E[y \mid x]}{\partial y}=\phi\left(\boldsymbol{\beta}^{\prime} \mathbf{x}\right) \boldsymbol{\beta}
$$

\section{Base de datos}

Utilizamos la Octava Encuesta de Innovación, que tiene por objetivo proporcionar información sobre la estructura del proceso innovativo en Chile y mostrar las relaciones entre este proceso y la estrategia de innovación de las empresas, el rendimiento económico, la capacidad de innovar y los factores que influyen en su capacidad para innovar. El diseño del formulario y la metodología que la encuesta utiliza sigue los parámetros generales sugeridos por la OCDE y aplicados por sus países miembros, también utiliza los lineamientos de la Community Innovation Survey (CIS) plasmados en el Manual de Oslo.

La población objetivo se compone de empresas naturales o jurídicas que desarrollen actividades dentro del país, las ventas anuales de estas deben ser superiores a $2.400 \mathrm{UF}$, razón por la cual no se incluyen microempresas. A nivel sectorial son consideradas las actividades más importantes que se desarrollan en Chile, sin embargo, la base utilizada no permite individualizar el sector de cada una de las empresas encuestadas. Los sectores considerados son: energía, minería, industria manufacturera, servicios de salud y sociales, hoteles y restaurantes, actividades inmoviliarias y empresariales, agricultura, construcción, comercio, pesca, transporte e intermediación financiera.

La encuesta de innovación es de corte transversal, se estudian los años 2011 y 2012, y con la totalidad de sus datos es representativa a nivel 
nacional. El tamaño de la muestra asciende a un total de $4.614 \mathrm{em}-$ presas. En tanto el total de empresas utilizadas en la investigación se reduce a 4.338 que son las empresas resultantes luego de verificar sus respectivos datos.

Realizamos diversos filtros a la base de datos de manera que el número total de empresas disponibles incialmente se redujo, en concreto, para la base utilizada en nuestra investigación eliminaron un total de 276 empresas, las cuales poseían errores en la digitación de datos. Específicamente 10 en el año de inicio de la empresa. 24 contaban con error en el monto de las exportaciones, donde estas eran superiores a las ventas totales del periodo. 219 empresas poseían ventas al 2012 inferiores a las 2.400 UF especificadas por la metodología de la encuesta, de este total 46 cuentan con ventas igual a cero. Finalmente 24 empresas fueron eliminadas por no poseer año de inicio de la producción y por ende la falta del dato de antigüedad de la firma. Hay que mencionar que las firmas de la muestra pertenecen a distintos sectores económicos y tienen distintas procedencias regionales. Dado el número de la muestra total se puede establecer que esta otorga resultados significativos y representativos estadísticamente. Se debe mencionar que los datos son de corte transversal y no representan una evolución o seguimiento a través de un periodo determinado de tiempo.

Del total de las observaciones analizadas, se observa que alrededor del $4 \%$ de las empresas tiene o es titular de algún derecho de propiedad intelectual o patente. Mientras que el $96 \%$ restante no ha solicitado ningún instrumento de propiedad en Chile ni en el extranjero. A continuación se muestran una tabla que presenta las principales características de las las empresas que sn sujeto de estudio.

Tabla 2. Descripción de las variables utilizadas

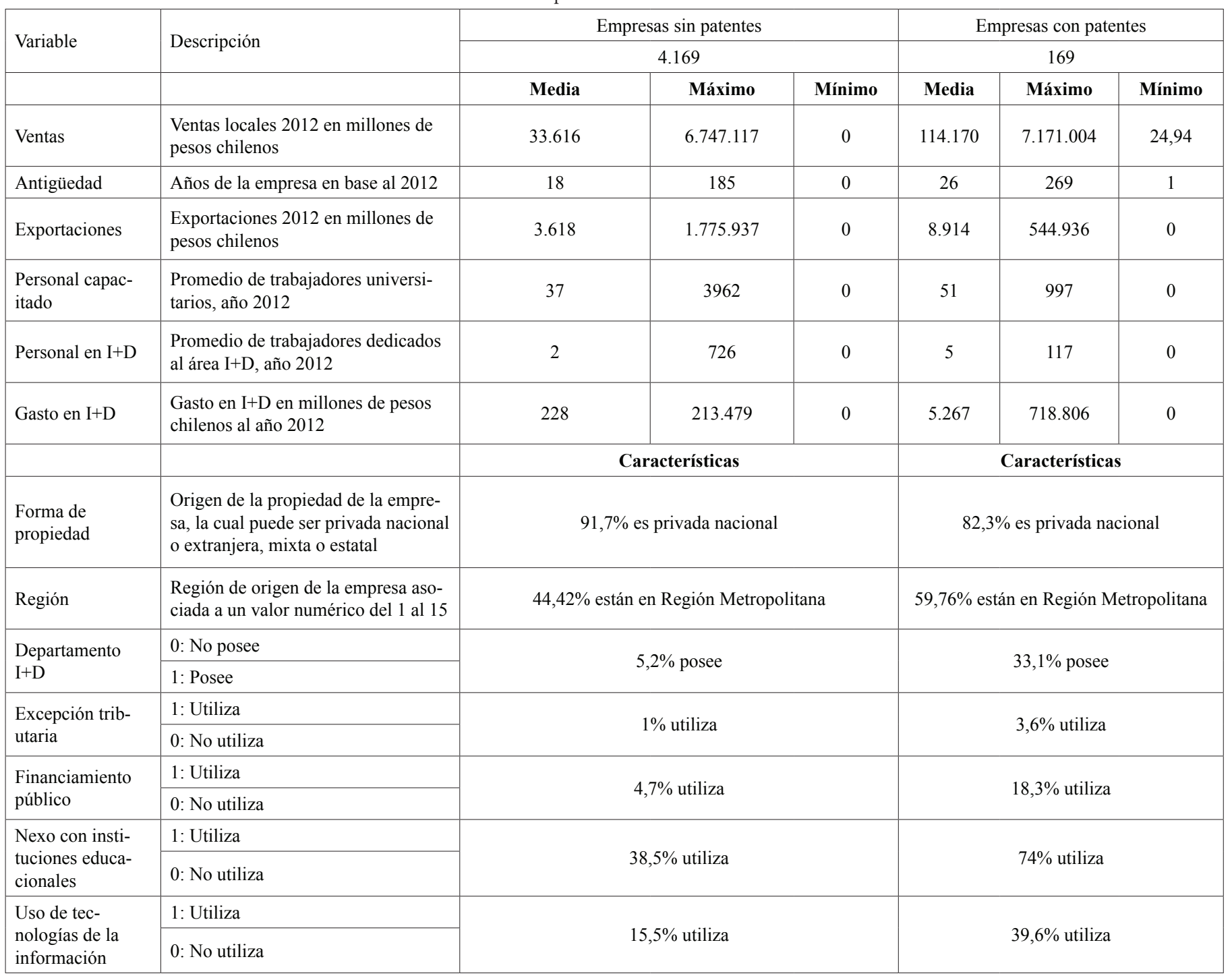

Fuente: Elaboración propia en base a datos de la encuesta de innovación 2011-2012. 


\section{Estimaciones}

Los resultados de las estimaciones se pueden observar en la tabla 3. Del total de las variables estudiadas, 7 presentan significancia. Personal capacitado, medido como el número de profesionales sobre el total de trabajadores de una empresa, Departamento I+D y Nexo con instituciones educacionales son estadísticamente significativas al 99\%, la Antigüedad, Uso de TI y Fondo público al 95\%, y finalmente la variable Región de los Ríos presenta significancia al 90\%. El resto de las variables no ha presentado significancia en sus coeficientes por lo que, para esta estimación, no influirían en la decisión de patentar en las empresas. Se observa que todas las variables que presentan significancia poseen un coeficiente con signo positivo, excepto la variable Región de los Ríos. Además, la significancia observada para las variables Nexo con Instituciones Educacionales y Uso de TI indican la relevancia que tienen los vínculos que puedan existir entre las empresas e instituciones de educación y/o tecnologías de la información, y como este vínculo influye en la creación de patentes.

En cuanto a las variables significativas, y considerando tanto el coeficiente como los efectos marginales de estas se puede aseverar que si la empresa posee un año más de antigüedad verá aumentada su probabilidad de poseer patentes en una proporción bastante pequeña. En tanto, al aumentar en uno la proporción de trabajadores capacitados (con profesión, magister o doctorado) sobre el total de trabajadores de la empresa aumenta la probabilidad de innovar en una proporción mayor. En cuanto a las variables dicotómicas, si la empresa utilizó fondos públicos su probabilidad de poseer patentes también aumenta. Asimismo, la variable relacionada al departamento I+D es la que posee el efecto marginal más importante de las variables analizadas, demostrando su importancia en el aumento de la probabilidad de patentar. El mismo caso anterior se repite tanto cuando la empresa posee nexos con entidades educacionales y si la empresa utilizó nuevas prácticas de organización de procesos (reingeniería de procesos, gestión de calidad etc.) como tecnologías de información pero con un efecto marginal menor. Por otro lado la variable Región de los Ríos presenta significancia, pero con un coeficiente y un efecto marginal negativo. Podemos interpretar esto diciendo que las empresas ubicadas en dicha región, por diversas razones o variables no observadas en este estudio, ven en desmedro su capacidad de innovación solo por el hecho de ubicarse en dicha Región. No obstante hay que considerar que los datos son variables dicotómicas, lo cual entrega información limitada, y dificulta una interpretación mayor. En relación a esto queremos señalar además que el foco de nuestro trabajo no es profundizar sobre diferencias espaciales.
Tabla 3. Coeficientes, significancias y efectos marginales de las variables

\begin{tabular}{|c|c|c|c|}
\hline Dimensión & Variable & Coeficiente $^{1}$ & $3 \mathrm{dy} / \mathrm{dx}$ \\
\hline \multirow{2}{*}{ Tamaño } & Ventas locales 2012 & $5,18 \mathrm{E}-08$ & $3,17 \mathrm{E}-09$ \\
\hline & Exportaciones 2012 & $-3,99 \mathrm{E}-07$ & $-2,45 \mathrm{E}-08$ \\
\hline Edad & Antigüedad & $0,0040023^{\star *}$ & 0,0002452 \\
\hline \multirow{2}{*}{$\mathrm{I}+\mathrm{D}$} & RRHH en I+D 2012 & $-0,000959$ & $-0,0000588$ \\
\hline & Gasto en I+D & $3,53 \mathrm{E}-06$ & $2,16 \mathrm{E}-07$ \\
\hline \multirow{3}{*}{$\begin{array}{l}\text { Forma de } \\
\text { propiedad }\end{array}$} & Propiedad nacional & $-0,3649418$ & $-0,0299417$ \\
\hline & Propiedad extranjera & $-0,1932427$ & $-0,010007$ \\
\hline & Propiedad mixta & $-0,4175191$ & $-0,0176454$ \\
\hline \multirow{14}{*}{$\begin{array}{l}\text { Región } \\
\text { geográfica }\end{array}$} & Región de Tarapacá & $-0,214838$ & $-0,0108515$ \\
\hline & Región de Antofagasta & $-0,3308421$ & $-0,150886$ \\
\hline & Región de Atacama & $-0,3327891$ & $-0,0150758$ \\
\hline & Región de Coquimbo & 0,0749469 & 0,0049141 \\
\hline & Región de Valparaíso & $-0,1425328$ & $-0,007751$ \\
\hline & Región O’Higgins & $-0,2429941$ & $-0,0120303$ \\
\hline & Región del Maule & $-0,2302869$ & $-0,0115401$ \\
\hline & Región del Biobío & $-0,3873302$ & $-0,0173516$ \\
\hline & Región de la Araucanía & $-0,2669446$ & $-0,0129454$ \\
\hline & Región de los Lagos & $-0,0504207$ & $-0,0029584$ \\
\hline & Región de Aisén & $-0,4116559$ & $-0,0172461$ \\
\hline & Región de Magallanes & $-0,3765633$ & $-0,016425$ \\
\hline & Región Metropolitana & $-0,1149965$ & $-0,0069739$ \\
\hline & Región de los Ríos & $-0,7728221^{*}$ & $-0,242683$ \\
\hline \multirow{2}{*}{$\begin{array}{l}\text { Base de } \\
\text { conocimiento }\end{array}$} & Departamento I+D & $0,6987536^{* * *}$ & 0,0219949 \\
\hline & Personal capacitado & $0,3590284^{* * *}$ & 0,0761969 \\
\hline \multirow{2}{*}{$\begin{array}{l}\text { Apoyo guberna- } \\
\text { mental }\end{array}$} & Exención tributaria & 0,0836733 & 0,0055475 \\
\hline & Fondo público & $0,3306041 * *$ & 0,0269454 \\
\hline \multirow{2}{*}{$\begin{array}{l}\text { Uso de infor- } \\
\text { mación }\end{array}$} & Uso de TI & $0,2079681 * *$ & 0,0146066 \\
\hline & $\begin{array}{l}\text { Nexo con inst. } \\
\text { educacionales }\end{array}$ & $0,291981 * * *$ & 0,019068 \\
\hline \multicolumn{2}{|c|}{ Constante $=-1,754555$} & \multicolumn{2}{|c|}{$y=0,02644641$} \\
\hline \multicolumn{2}{|c|}{ Pseudo R2 = 0.1393} & \multicolumn{2}{|c|}{ Chi2 $=196,30$} \\
\hline
\end{tabular}

Fuente: Elaboración propia en base a los resultados del análisis.

\section{Resultados y discusión}

El tamaño de la empresa no presentó significancia para nuestro caso. Recordemos que Buesa \& Molero (1996) señalaron que el tamaño de una firma está positivamente relacionado con que la empresa emprenda actividades innovadoras, ellos utilizaron también una base de datos de corte transversal, pero considerando una menor cantidad de variables en su estudio. Nosotros no podemos corroborar aquello con nuestros resultados, pero podemos explicar las diferencias entre ambos debido a la disparidad de variables utilizadas.

Murillo \& Hill de Tito (2006) establecen que una empresa que exporta se ve influenciada a cumplir con estándares más altos y por ende a innovar, podemos mencionar que su estudio se centra en un determinado sector industrial, no considerando a todos los sectores en su conjunto. En nuestro caso, nuestra base considera variadas industrias. Nuestras estimaciones no tuvieron efectos significativos respecto al nivel de exportaciones.

Donde ${ }^{*} \mathrm{p}<0.1^{* *} \mathrm{p}<0.05^{* * *} \mathrm{p}<0.01$ 
La antigüedad de la empresa posee significancia y un efecto positivo, por lo que podemos comprobar lo expuesto por Buesa \& Molero (1996) quienes postularon que la edad de la firma aumenta la capacidad innovadora de la empresa.

En tanto, las variables relacionadas a la forma de propiedad, éstas no presentaron significancia en ninguno de sus tipos, por lo que no es posible comprobar en nuestro estudio que las empresas que poseen propiedad extranjera poseen mayores influencias para patentar, lo que ha sido expuesto por estudios de Buesa \& Molero (1992) y Álvarez \& García (2012), para este último estudio podemos acotar que sólo se considera el sector manufacturero.

No pudimos comprobar que la localización de la empresa influya en la probabilidad de patentar. Reconocemos que nuestra base de datos no es idónea para aislar este efecto espacial. Recordemos que medimos la localización como la ubicación de una empresa en una determinada región del país, esta medida es poco precisa dado que no nos permite conocer coordenadas de varias empresas dentro de una misma región. El 2,4\% $\left(0,04^{\star} 0,6\right)$ del total de empresas patenta y se encuentra en la Región Metropolitana.

Respecto a la base del conocimiento, podemos explicar esta por dos variables profesionales sobre el total de trabajadores de la empresa y la existencia de un departamento I+D. Los resultados van en la línea de los estudios de Vega et al (2005), observando una relación positiva entre estas variables y la innovación de la empresa. En este contexto, Álvarez \& García (2012) encontraron una relación positiva entre las competencias del recurso humano y la innovación, pero no encontraron evidencia de la misma relación para la existencia de un departamento de $\mathrm{I}+\mathrm{D}$ y su relación con la innovación. Es importante mencionar que en dicho estudio, la innovación es medida en base a dos variables distintas; la primera es una variable binaria que toma valor 1 si la firma gasta en innovación, mientras que la segunda se refiere al esfuerzo innovador, el cual es medido como el logaritmo de la inversión promedio por trabajador que realiza la empresa en adquirir tecnologías de capital, gestión, capacitación tecnológica e I+D.

Otra variable significativa se refiere a los instrumentos gubernamentales de apoyo a la I+D. Nuestros resultados indican que el financiamiento público es significativo y su efecto es positivo, por lo que podemos comprobar la importancia dada por Teubal (1996) a este instrumento y el beneficio que genera en la innovación empresarial.

Por otro lado, no comprobamos que los incentivos tributarios sean determinantes para las actividades innovadoras de las empresas. Esto no necesariamente se debe a que esta no influya en la innovación, sino que puede ser explicado por el desconocimiento de esta ley por parte de las empresas y/o porque su efecto puede estar subvalorado. De acuerdo a datos obtenidos de la encuesta de innovación, el 29\% de las empresas conoce la ley, y sólo el 1,2\% de empresas la utilizan. El uso de tecnologías de la información presentó significancia y un signo positivo en su derivada, lo que va en una misma línea que lo expuesto por Martínez \& Pérez (2001), demostrando la importancia de dicha variable para el patentamiento de las empresas.

\section{Recomendaciones}

A nivel empresarial la innovación es baja, en la muestra solo el $4 \%$ de las empresas posee patentes, por lo que para mejorar esta situación es de suma importancia que surgan políticas públicas orientadas a la generación de conocimiento y tecnologías en las firmas. Considerando los resultados de nuestro estudio podemos desarrollar posibles líneas de actuación, tales como el desarrollo de nuevos y mayores incentivos gubernamentales, y la masificación de las exenciones tributarias de manera que una mayor cantidad de empresas accedan. En definitiva, es recomendable el desarrollo de nuevos formas de apoyo que estimulen a las empresas a crear patentes de invención.

Respecto a la proporción de personal capacitado, actualmente el Estado otorga distintas becas para que profesionales se especialicen, política que es coherente con los resultados obtenidos en la investigación, sin embargo no es una práctica lo suficientemente masificada, quizás debido el alto costo asociado. Es por esto que una posible alternativa es que el Estado incentive a que las propias empresas capaciten a sus trabajadores, por medio de las mismas excenciones tributarias, lo que disminuiría en gran cuantía el costo directo que el Estado desembolsaría.

\section{Agradecimientos}

Queremos agradecer a los alumnos Matías Norambuena Suárez y Edgard Seguel Sáez de la carrera de Ingeniería Comercial de la Universidad de Concepción campus Chillán, por su aporte en la revisión de literatura, dicho aporte fue un importante insumo en la elaboración del presente documento.

Agradecemos el financiamiento aportado por la Vicerrectoría de Investigación y Desarrollo (VRID) de la Universidad de Concepción. Código 215.097.013-1.0IN

Finalmente agredecemos al Ministerio de Economía, Fomento y Turísmo, y al Instituto Nacional de Estadísticas (INE) quienes pusieron a nuestra diposición la base de datos de la Octava Encuesta de Innovación en Empresas.

\section{Referencias}

Álvarez, E., \& García, W. (2012). Determinantes de la Innovación: Evidencia en el Sector Manufacturero de Bogotá. Semestre Económico. Volumen 15, N³2, 129-160.

Belussi, F., \& Arcangeli, F. (1998). A typology of networks: Flexible and Evolutionaty Firms. Research Policy, vol. 27(4). 415-428. DOI: http://dx.doi.org/10.1016/S0048-7333(98)00074-2

Benavente, J. M. (2005). Investigación y desarrollo, innovación y productividad: un análisis econométrico a nivel de la firma. Estudios de economía, 39-67.

Bronwyn, H., \& Van Reenen, J. (2000). How effective are fiscal incentives for R\&D? A review of the evidence. Research Policy, vol 29 , 449469. DOI: http://dx.doi.org/10.1016/S0048-7333(99)00085-2 
Buesa, M., \& Molero, J. (1992). Capacidades tecnológicas y ventajas competitivas en la industria española: Un análisis a partir de las patentes. Ekonomiaz $N^{\circ} 22$, 220-247.

Buesa, M., \& Molero, J. (1996). Tamaño empresarial e innovación tecnológica en la economía española. Documentos de trabajo del IAIF; $n^{\circ} 1,1-21$.

Cohen, W., \& Levin, R. (1989). Empirical studies of innovation and market structure. En Handbook of industrial organization (págs. 1059-1107). DOI: http://dx.doi.org/10.1016/S1573-448X(89)02006-6 Díaz, M. (2008). La propiedad industrial y los sistemas de patentes en el mundo de la información. ACIMED.

Fernández, S. Otero, L. Rodeiro, D. \& Rodríguez, A. (2009). Factores determinantes de la creación de spin-offs universitarias. Revista europea de dirección y economía de la empresa. Vol. 19 Nº 1. 47-68.

Forero, C., Laureiro, D., \& Marín, A. (2011). Innovation Patterns and Intellectual Property in SMEs of a developing country. Innovar. Vol 21 N²4. Galeras de Administración, 113-127.

Greene, W. (1999). Análisis econométrico. Prentice Hall.

Hägerstrand, Torsten (1953). Innovation Diffusion as a Spatial Process, Chicago University Press, Chicago, Estados Unidos.

Hernández, C., \& Gonzáles, D. (2013). Estudio de la Evolución Tecnológica en Empresas de Celulares a Partir de la Actividad de Patentamiento. Journal of Technology Management \& Innovation, Vol 8, 161-177. DOI: http://dx.doi.org/10.4067/S0718-27242013000300069

Juliao, J., Barrios, F., Schmutzler, J., \& Sánchez, I. (2013). Relación entre la estrategia de innovación de la firma y su decisión de patentar: evidencia de empresas pertenecientes al sector manufacturero colombiano. Estudios Gerenciales, vol 29, 313-321. DOI: http://dx.doi. org/10.1016/j.estger.2013.09.005

Martínez, Á., \& Pérez, M. (2001). Patentes y desarrollo regional de instrumentos médicos en la industria española. Economía Industrial. $N^{\circ} 338,147-155$.

Murillo, D., \& Hill de Titto, P. (2006). Los Determinantes de las Actividades Tecnológicas en México. Obtenido de Programa de Ciencia y Tecnología del Centro de Investigación y Docencia Económicas: www.cidecyt.org
OCDE. (2009). Recuperado el 21 de Marzo de 2015, de Manual de Estadísticas de Patentes de la OCDE: http://www.oepm.es/comun/ documentos_relacionados/Publicaciones/monografias/manualEstadisticas.pdf

OCDE. (2015). Main Science and Technology Indicators. Recuperado el 20 de Marzo de 2015, de http://www.keepeek.com/Digital-AssetManagement/oecd/science-and-technology/main-science-and-technology-indicators/volume-2014/issue-2_msti-v2014-2-en\#page31

Rothwell, R., \& Dogson, M. (1994). Innovation and size of firm. The Handbook of Industrial Innovation (págs. 310-324).

Salgado, H., \& Chovar, A. (2010). ¿Cuánto Influyen las Tarjetas de Crédito y la Deuda Hipotecaria en el Sobre Endeudamiento de los Hogares en Chile. Serie Documento de Trabajo EconUdeC 12, 1-27.

Salmi, P., \& Torkkeli, M. (2009). Inventions Utilizing Satellite Navigation Systems in the Railway Industry - An Analysis of Patenting Activity. Journal of Technology Management \& Innovation, Vol $4 \mathrm{~N}^{\circ} 3$, 46-58. DOI: http://dx.doi.org/10.4067/S0718-27242009000300005

Sancho, R. (2002). Indicadores de los Sistemas de Ciencia, Tegnología e Innovación. Economía Industrial, 97-109.

Teubal, M. (1996). R\&D and technology policy in NICs as learning processes. World development, 449-460.

Tseng, A., \& Raudensky, M. (2014). Performance Evaluations of Technology Transfer Offices of Major US Research Universities. Journal of Technology Management \& Innovation, Vol 9 N¹, 93-102. DOI: http://dx.doi.org/10.4067/S0718-27242014000100008

Vega, J., Fernández, I., Gutiérrez, A., \& Manjarres, L. (2005). Los Determinantes de la Innovación Tecnológica en la Empresa: Una Aproximación A Través del Concepto de Capacidad de Absorción. XI Semario Latino-Iberoamericano de Gestión Tecnológica, (págs. 1-18). Salvador.

Vilalta, C., \& Banda, H. (2008). Dos pruebas de hipótesis sobre la geografía de las patentes en México. Economía, Sociedad y Territorio. Vol. VII. $\mathrm{N}^{\circ} 28,1007-1032$. 